\title{
WHAT HAPPENS TO BINOCULARITY IN PRIMATE STRABISMUS?
}

\author{
RONALD G. BOOTHE and RICK J. BROWN \\ Atlanta, Georgia
}

\begin{abstract}
SUMMARY
Normal humans for whom the positions and movements of the two eyes are constrained to be yoked together are able to extract rich binocular sensory information from the environment. Humans with strabismus are deficient in extracting some of this information. Studies of strabismus in non-human primates can augment what has been learned from humans about relationships between strabismus and sensory binocular function. For example, speculation about the role of binocular vision in primate evolution can help us understand why it is that the advantages of sensory binocular function outweigh the disadvantages of having the positions of the two eyes yoked together. Physiological optics assessments of fixation patterns and accommodative responses in monkeys provide information about how the brain accomplishes and coordinates motor and sensory binocular functions, and sets the stage for determining underlying neural mechanisms responsible for this coordination. Finally, a developmental perspective, concerned with events that occur during an early sensitive period in the life span of an infant primate, can help us understand how nature and nurture interact to set up this complex neural system in normal individuals, and how this process is disrupted in conditions such as strabismus.
\end{abstract}

\section{BINOCULAR FUNCTION EVALUATED FROM A PSYCHOBIOLOGICAL PERSPECTIVE}

Imagine that someone were to clasp your wrists into a set of handcuffs. As a result you would find that the positions of your left and right hands were constrained to be near each other and the movements of your hands would be yoked together. For example, if you wanted to move your left hand to the left side of your body to pick up an object, you would be forced to simultaneously also move your right hand to the left side. It is unlikely that you would consider this

Correspondence to: Dr Ronald G. Boothe, Division of Visual Science, Yerkes Regional Primate Research Center, Emory University, Atlanta, GA 30322, USA. yoking to be a functional advantage! Why then should we consider individuals with a strabismus (i.e. conditions in which the two eyes do not always aim in the same direction) to have a visual defect, and 'normal' individuals in whom the two eyes are effectively yoked together, to be at a functional advantage? The answer, from the point of view of evolution, is likely to be that there are sufficient functional advantages to having binocular sensory processing to overcome the impediments to binocular motor control that come from yoking the eyes together.

The binocular visual system in primates consists of two inter-related and reciprocally interacting subsystems that involve sensory and motor processing (Fig. 1). This system is exceedingly complex in terms of both its computational requirements and its neural circuitry, and its operation depends upon an exquisite coordination of binocular motor control and sensory processing. ${ }^{1-5}$ But what does it provide in terms of new or improved visual function? It is commonly assumed that the functional advantage that is obtained from binocular processing involves some aspect of depth perception. There can be little doubt that it is important for primates to be able to determine the locations of objects in three-dimensional space relative to themselves, and in particular to be able to detect approaching objects. However, much of the rich information about positions and movements of objects relative to an observer can, in principle, be carried out by appropriate processing of monocularly available information. ${ }^{6-9}$ Thus, when trying to construct arguments about the advantages that accrue to an organism that has binocular vision, it is not sufficient to demonstrate that binocular processing is able to achieve some particular function. We also need to address the issue of whether achieving a particular function via binocular processing has some advantageous characteristics over and beyond those which can be obtained from analysing monocular information. 


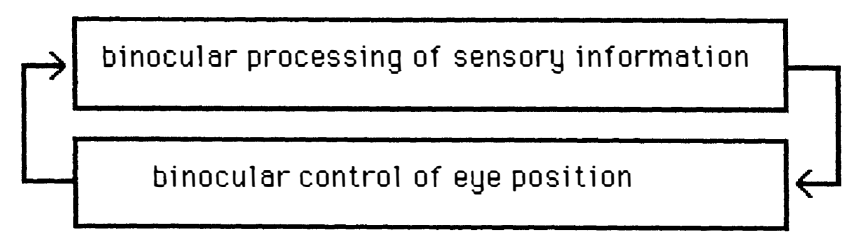

Fig. 1. Binocularity involves reciprocal interactions between two subsystems that perform motor and sensory processing. These two subsystems are exquisitely interdependent such that the function of each can only be fully appreciated by examining how its own processing relates to the function of the overall binocular system. Constraints that are present within a single subsystem, such as the fact that the eyes must be yoked together during motor control, are understandable when it is understood that these constraints are necessary for the overall system to function properly.

Over evolutionary time, binocular vision was probably accomplished in a stepwise fashion, building on earlier, simpler functions. ${ }^{10,11}$ For example the eyes of some species that had strictly monocular processing of non-overlapping visual fields may have begun to move towards the front of the head due to a chance genetic mutation. Now the animals of this species had the potential of fixating a single object with both frontal eyes simultaneously. At this point, converging the eyes on the same object could be accomplished by independent monocular movements, but the stage would have been set for a more efficient system of motor control - a system in which convergence was achieved with a single mechanism involving feedback from simultaneous sensory processing of information coming from each fovea. Such a system would provide rudimentary motor fusion.

Given the above scenario, the emergence of disparity processing would have augmented the efficiency of motor fusion when changing fixation from objects at different distances. The computations needed to make quick and accurate eye movements to objects inside (crossed disparity) and outside (uncrossed disparity) the horopter could be provided by rudimentary sensory processing of simultaneous information coming from each retina.

The presence of this kind of primitive disparity processing does not necessarily imply a perception of depth as we understand it, but might simply involve registering shifts in retinal correspondence. The perception associated with this disparity processing might very well have involved only diplopia or suppression prior to the time when depth-perception evolved. The subsequent emergence of sensory fusion, including a percept of depth, from disparity processing would have both augmented the effectiveness of, and simplified, motor fusion, because an immediate depth percept from two disparate monocular images is probably more efficient to access and interpret than learning to associate each pair of raw disparities with their proper distances.
In addition to static disparity, binocular vision also encompasses binocular motion processing (see Fig. 2). This form of binocular processing very probably had its evolutionary roots at a time before eyes even started to move towards the front of the head to work better together. Motion perception in itself is probably more primitive than binocularity: the toad, for example, whose prey-catching ability relies on its perception of motion, seems oblivious to static stimuli, and does not have the capacity for binocular disparity processing. ${ }^{12-14}$ Moreover, relative motion among objects that is induced by selfmotion can be used to detect relative distances by motion parallax. This latter phenomenon was first recognised as a cue to depth by Helmholtz, ${ }^{15}$ and has now been firmly established to give information about the three-dimensional structure of objects. ${ }^{16}$ It is also known that motion parallax can be exploited by animals that have no binocular vision as a means to estimate distance to a prey. For example, some insects can locate a prey with precision by moving the head from side to side before striking the target, thus

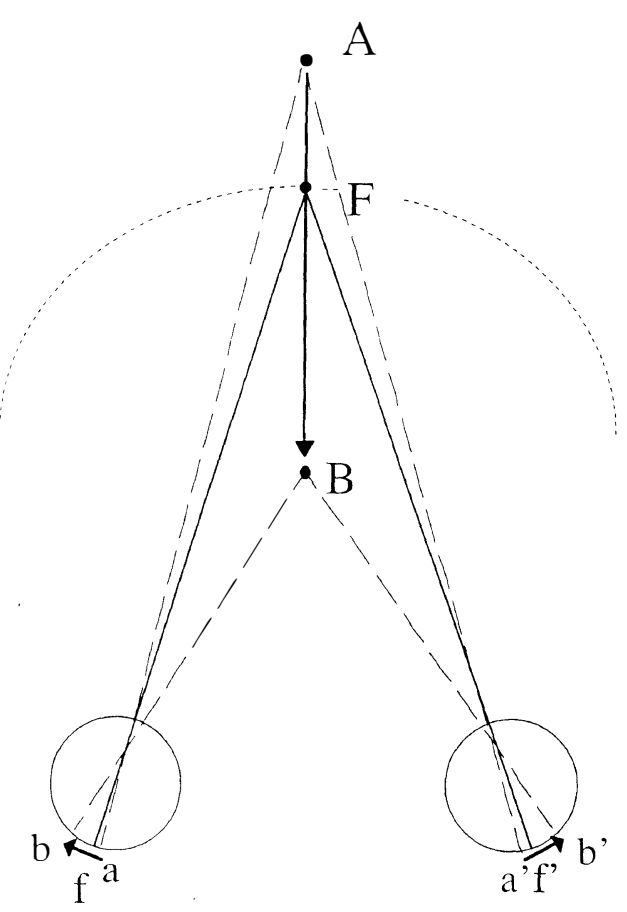

Fig. 2. When an object changes in depth relative to a binocular observer, there is information available in the optic array that can be obtained by binocular processing of information about relative positions in the two eyes (binocular disparity) and relative motions in the two eyes (binocular motion). One of the hallmarks of primate binocular visual systems is the efficiency with which they combine processing of these two kinds of binocular information. This figure illustrates what happens in the retinal images of the left and right eyes when an object moves from point A which is beyond the fixation point to point $\mathrm{B}$ which is closer than the fixation point. The retinal disparity for position changes, and the monocular motions generated in the two eyes are in the opposite direction. 
making up for the lack of static stereopsis by extracting the same disparity information over time. ${ }^{17}$ Since movement of the self is required for such a feat, it is possible that the emergence of static stereopsis in predators served the functional advantage of allowing an estimation of the distance to the prey while remaining still, and therefore inconspicuous. This functional role may have helped maintain static stereopsis in canine species, such as cats, but is unlikely to have played a large functional role in primates.

A more plausible role for a functional advantage of binocular sensory processing in primates involves stereomotion. Primate visual systems are able to utilise both binocular disparity and binocular motion information to achieve perception of motion-in-depth in which an observer is aware of the trajectory of objects that are approaching or receding in threedimensional space. ${ }^{16}$ Motion-in-depth can be specified by several different kinds of information simultaneously, some of which can be picked up while viewing with only one eye (e.g. changes in retinal image size). We will restrict our use of the term stereomotion to refer to the perception of motion-in-depth that can only occur under binocular viewing conditions. Stereomotion can be demonstrated in a laboratory setting by using special displays in which all monocular cues are eliminated.

Fig. 2 illustrates the geometrical layout of an object that moves from position $A$ to position $B$ with respect to an obsever who is fixating binocularly at fixation point $F$. The object is moving towards the nose of the subject. The images projected from the fixation point onto the fovea of each retina are labelled $f$ and $f^{\prime}$ for the left and right eye respectively. The projections of the object's images onto each retina when the object is at position $A$ and at position $B$ are indicated by the dashed lines. Position $A$ lies outside the plane of fixation and projects onto non-corresponding points ( $a$ and $a^{\prime}$ ) that have uncrossed disparity. At point $B$, the object has moved towards the head, such that it projects onto non-corresponding pøints $\left(b\right.$ and $\left.b^{\prime}\right)$ that have crossed disparity. Stereomotion information can be picked up by processing these changes in binocular disparity over time. However, note that the object moving towards the nose in Fig. 2 also results in monocular motion to the right in the right eye and, simultaneously, monocular motion to the left in the left eye. Thus, stereomotion information can also be picked up by binocular processing of this relative motion in the two eyes. The geometry associated with binocular motion processing for the more general case in which objects move in any direction relative to the fixation point in the horizontal plane has been worked out by Poggio and Talbot. ${ }^{18}$ They demonstrated that binocular motion processing can, in principle, provide quantitative as well as qualitative information about direction of object motion.

Primate visual systems might, in principle, have evolved two separate binocular processing systems for achieving stereomotion: one that makes use of binocular disparities at different disparity planes, and a second that involves binocular synthesis of the different speeds and directions of monocular motion. However, there is evidence that, at least in humans, individuals are able to pick up stereomotion information that does not depend on processing of either binocular disparity or the binocular synthesis of monocular motion. This was demonstrated psychophysically by Shimojo et $\boldsymbol{a l}^{19}{ }^{19}$ in an elegant study in which observers viewed moving objects through a small aperture that was positioned close to the observer. The viewing situation is illustrated to Fig. 3. Consider an object that is moving along a trajectory from position $A$ to position $C$. At time $t 1$ this object is seen at position $A$ by the right eye. However, it is invisible to the left eye at this time. At time $t 2$ this same object is seen at position $C$ by the left eye but is invisible to the right eye. What we traditionally think of as binocular disparity processing cannot provide any information about stereomotion for this object because there is no point in

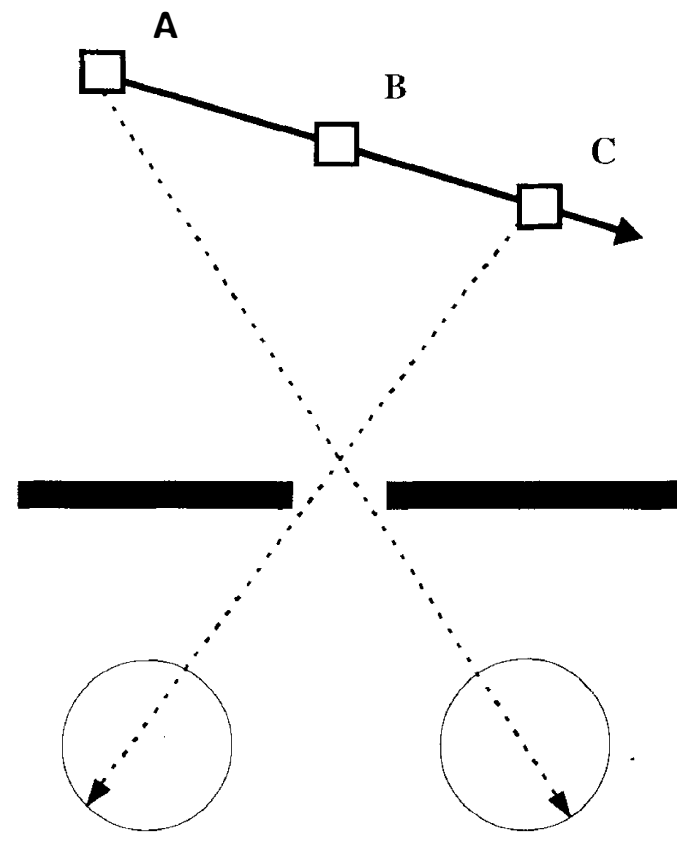

Fig. 3. When an observer views a moving object through a small aperture, there is no information that can be obtained about depth by doing exclusively disparity processing because there is no time when the object forms simultaneous images in both eyes. This is illustrated by an 'object that moves from point $\mathrm{A}$ at time $\mathrm{t} 1$ to point $\mathrm{C}$ at time $\mathrm{t} 2$. There is no binocular disparity present at either $\mathrm{t} 1$ or $\mathrm{t} 2$. Similarly, there is no simultaneous motion generated in both eyes at either $\mathrm{t} 1$ or $\mathrm{t} 2$. In order for a binocular visual system to pick up the trajectory of this object it must do complicated binocular processing of information across 'space-time'. 
time at which the two eyes receive simultaneous images from the object that can be analysed for binocular disparity. Similarly, there is no point in time during which there is simultaneous motion produced by this object in the two eyes that can be analysed for binocular synthesis of monocular motion. Shimojo et al. ${ }^{19}$ summarise their results by stating that it is not sufficient for the stereomotion processing system to process information over time while holding position constant (local motion processing) or information over space while holding time constant (static disparity). Instead, stereomotion processing needs to interpolate over 'space-time'. The complexities of the processing required for any binocular visual system to achieve stereomotion perception under these conditions are rather remarkable. Nevertheless, human observers are able to perceive stereomotion under these conditions. Are there conditions in the environment in which primates evolved that might have made such a complex system of binocular stereomotion processing advantageous?

Over evolutionary time, primates were confronted with avoiding approaching predators while spending considerable time eating fruits and leaves in a leafy forest environment. The view through the foliage surrounding the head in such an environment will produce the optical equivalent of looking at the surrounding space through a number of small apertures. It does not seem unreasonable to speculate that the ability to pick up information about approaching predators under these conditions would provide a strong functional advantage to primates, perhaps an advantage that substantially outweighed the disadvantages associated with forcing the eyes to be yoked together.

If this speculation is correct, then species in which binocular processing evolved in other kinds of environments may not have these same stereomotion properties. For example, these special properties of stereomotion would probably not confer any particular advantage for species that evolved as predators in a more open environment. It is not obvious that these advantages continue to offer a particularly strong functional advantage to modern-day human primates either, and this could be one factor that accounts for the increased incidence of strabismus in humans compared with non-human primates (see Boothe ${ }^{20,21}$ for discussion of other factors).

Speculations about other possible functions for a stereomotion perception have been put forth based on psychophysical studies of humans. Beverly and Regan ${ }^{22,23}$ have argued that stereomotion provides an accurate means of avoiding objects that are about to hit the head. Their arguments are based on the following evidence: (1) different psychophysical mechanisms are used for processing object motion passing to the right or to the left of the head; (2) sensitivity for object motion towards the head is particularly sensitive. These abilities would obviously aid an individual in detecting and avoiding the impact of approaching objects that are on or near a collision course with the head. Richards ${ }^{24}$ has argued that stereomotion processing could also provide a very efficient signal for controlling vergence eye movements to track moving objects.

\section{PHYSIOLOGICAL OPTICS ASSESSMENTS OF FIXATION AND ACCOMMODATION}

Until the early 1980s there were no reports of naturally occurring strabismus in monkeys. This lack of widespread occurrence of strabismus in monkeys seemed surprising given the general similarities of human and monkey visual systems, and led Jampolsky ${ }^{25}$ to speculate that there must be something fundamentally different about the monkey oculomotor system that makes it impervious to the conditions that lead to misalignment in human infants. However, an extensive screening programme of every infant born at the breeding colony of the Regional Primate Research Center at the University of Washington over a period of several years identified a number of monkeys with naturally occurring infantile strabismus. ${ }^{26,27}$ Ten of these animals have undergone extensive study using physiological optics methods to determine whether or not the characteristics of their strabismus mimic common syndromes of human infantile 'strabismus. ${ }^{28,29}$ Two of the monkeys were determined to have a pattern of oculomotor deficits comparable to the human syndrome of essential infantile esotropia. Five monkeys had a non-paralytic esodeviation with an angle greater at near than at distance and were classified as having a condition similar to human early-onset accommodative esotropia. An eighth monkey had bilateral haemorrhages of the anterior chamber at birth followed by lens opacities that persisted for several months. The esotropia in this monkey was classified as being induced by early visual deprivation. In a ninth monkey the strabismus changed from an esotropia in infancy to an exophoria in adulthood, and in a final monkey an esotropia in infancy resolved to an orthophoria in adulthood.

Examination of accommodative responses in these monkeys revealed several important findings about the characteristics of binocular control of the near reflex. ${ }^{29}$ Accommodation was always accurate under monocular viewing conditions, with the exception of the inability of one myopic eye to focus distant targets. The accommodative response in the two eyes was always consensual, under both monocular and binocular viewing conditions. Thus, in cases where there was an anisometropia, one eye was always focused accurately at the target distance and the 


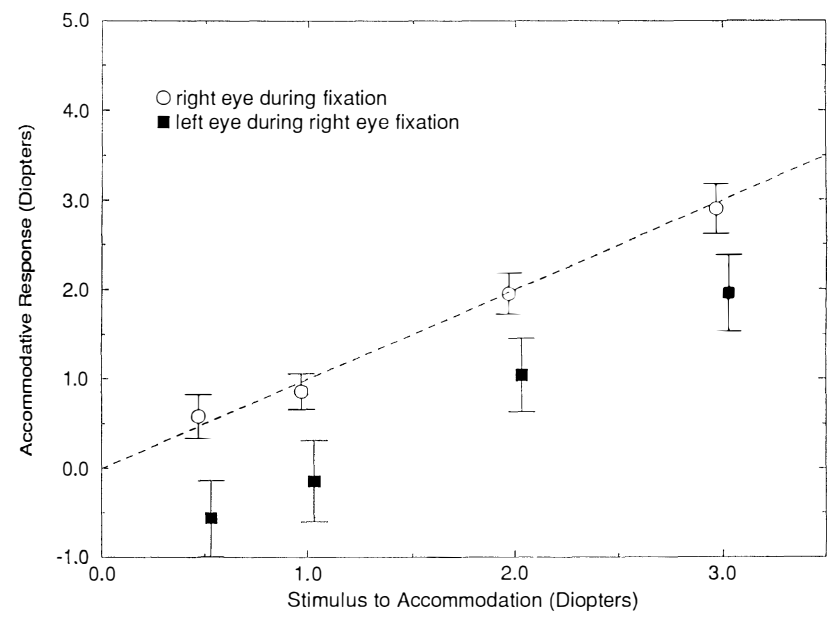

Fig. 4. The accommodative response is shown as a function of the accommodative stimulus. An observer with perfect focus would be expected to generate data points that fall along the dashed line. The results shown here were obtained from a monkey with an early onset accommodative esotropia. This monkey had a mild amblyopia in the left eye and always fixated with the right eye. This monkey also had an anisometropia of about 1 dioptre. The figure demonstrates that this monkey always accommodates accurately with the right eye and that accommodation is always consensual. As a result the left eye is always in error by about 1 dioptre.

fellow eye was in error by an amount predicted by the magnitude of the anisometropia. A methodology in which we recorded fixation and accommodation simultaneously revealed that the eye that was controlling fixation was also the eye that controlled accommodation. This is illustrated for one of our monkeys with an accommodative strabismus in Fig. 4. Accurate accommodation would be expected

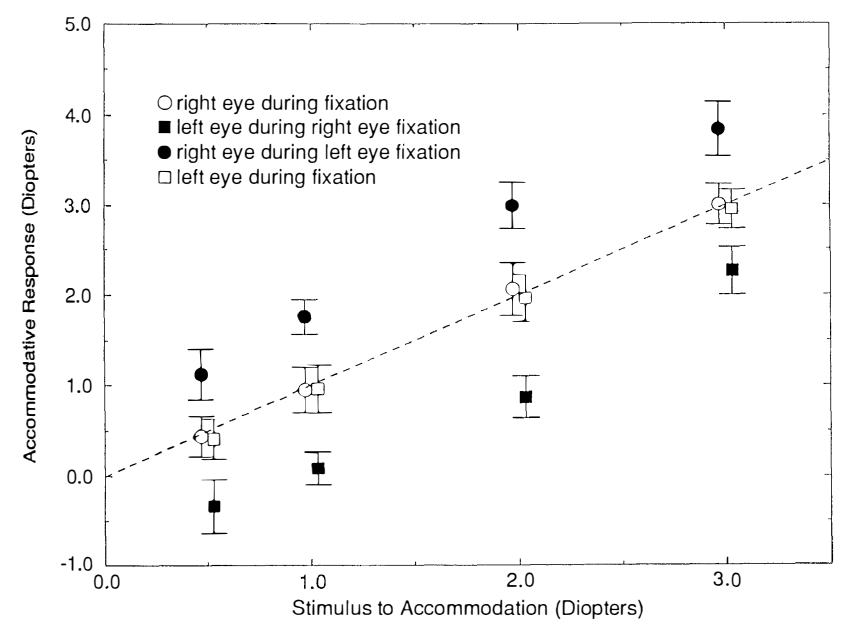

Fig. 5. The accommodative response is shown as a function of the accommodative stimulus as in Fig. 4. This figure shows results for a monkey that has an essential infantile esotropia. This monkey alternates fixation and has an anisometropia of about 0.75 dioptre. Simultaneous measurements of fixation and accommodation revealed that the eye which was fixating was the eye with accurate accommodation while the non-fixating eye was always the eye with inaccurate accommodation. to fall along the dashed line in this figure. This monkey had a mild strabismic amblyopia in the left eye and always used its right eye for fixation. The right (fixating) eye always exhibits accurate accommodation, and the left eye is always in error by an amount that is predictable by the magnitude of anisometropia that is present in this animal.

Similar measurements on animals which alternate fixation revealed that control of the near reflex alternates with alternation of fixation. This is revealed in Fig. 5 in which accommodative results are presented for one of our monkeys with an essential infantile esotropia. This monkey alternates fixation and simultaneous measurements of fixation and accommodation revealed that the eye which was fixating the target was always the eye with accurate accommodation. When fixation switched, so did accommodation, with the net result that one eye was always accommodated accurately and the fellow eye was always in error by an amount predicted by the anisometropia present in this animal.

Results from a monkey with an exophoria strongly suggest that only one eye is in control of the near reflex at any one moment, even when both eyes are fixating. This is illustrated in Fig. 6. Notice the similarity in the pattern of results shown in Figs. 5 and 6. In Fig. 5, there was an alternation in fixation and this allowed us to demonstrate that it was always the fixating eye that was in control of accommodation. However, in Fig. 6, both eyes are always fixating

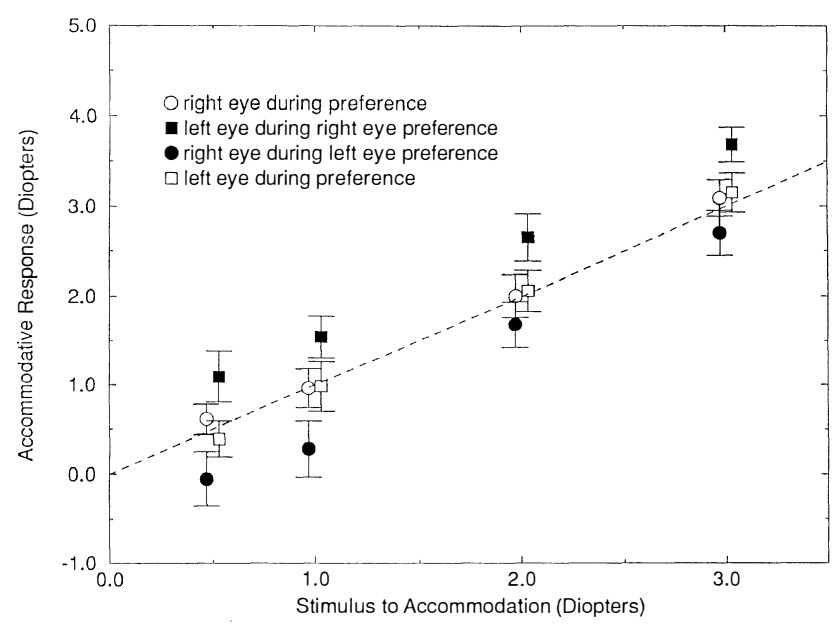

Fig. 6. The accommodative response is shown as a function of the accommodative stimulus as in Figs. 4 and 5. This figure shows results for a monkey that has an exophoria. This monkey has an anisometropia of about 0.5 dioptre. Comparison of the results in Figs. 5 and 6 reveals a similar pattern. At any one accommodative distance there is a bimodal distribution of responses in which one eye is always accurate and the fellow eye is inaccurate. However, in this monkey there is no tropia so we cannot differentiate the fixating from the non-fixating eye. The similarity of the pattern of results to those seen during alternating fixation in Fig. 5 leads us to conclude that one eye (designated here as the preferred eye) is in control of accommodation at any one time and that the preferred eye alternates over time. 
the target under binocular conditions, so we have no way to differentiate objectively between the fixating and non-fixating eyes. Nevertheless, the similarity in the pattern of results seen in the two animals leads us to conclude that only one eye is in control of the near reflex at any one moment even under conditions in which fixation is binocular.

These results are consistent with a simple model of binocular oculomotor control illustrated schematically in Fig. 7. There are several important features of this model. Note that only one eye is in control of fixation at any one moment in time, and it is sensory input from this eye that controls the position of the fixating eye. In contrast, sensory input from the nonfixating (fellow) eye does not control the position of the fellow eye. Instead, sensory inputs from both the fixating and fellow eyes enter a stage of binocular processing ('double eye' processing, borrowing the terminology used by Hering ${ }^{30}$ ). The results from the accommodative studies just presented demonstrate that the same eye that is in control of fixation is also in control of accommodation. Thus, the binocular sensory system receives accommodative input from the fixating eye that can be used for generating accommodative vergence and receives sensory input from both eyes that can be used for generating disparity vergence. The output of this binocular sensory processing, represented by the arrow that passes out of the 'double eye' processing box in Fig. 7, controls the final position of the fellow eye and represents motor fusion.

These findings place important constraints on the properties of the neural mechanisms that control the near reflex. Extensive studies of the neural mechanisms of vergence and accommodative control in monkeys have emphasised midbrain structures that are closely associated with oculomotor output. ${ }^{31,32}$ Relatively little is known about the neural pathways that provide sensory input to these structures. The

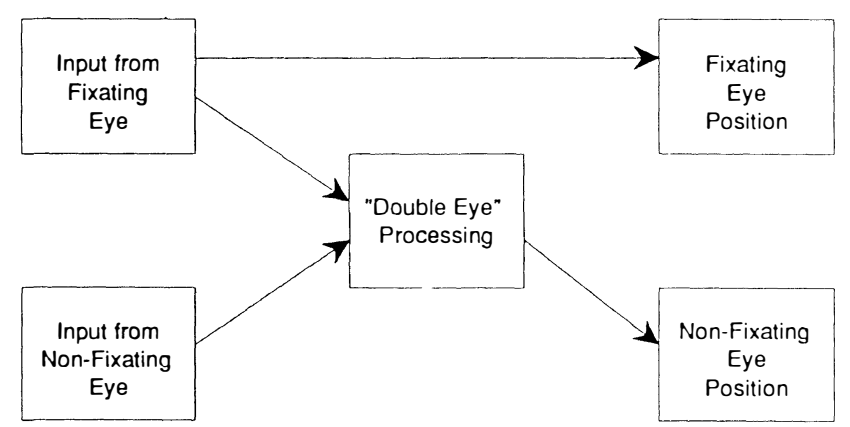

Fig. 7. A simple model for controlling motor fusion. The input from the fixating eye controls the position of the fixating eye and also controls accommodation. The position of the fellow eye is controlled by binocular processing (labelled as 'Double Eye' Processing). The binocular processing system receives accommodative information from the fixating eye to be used in generating accommodative vergence, and disparity input from both eyes to be used in generating disparity vergence.

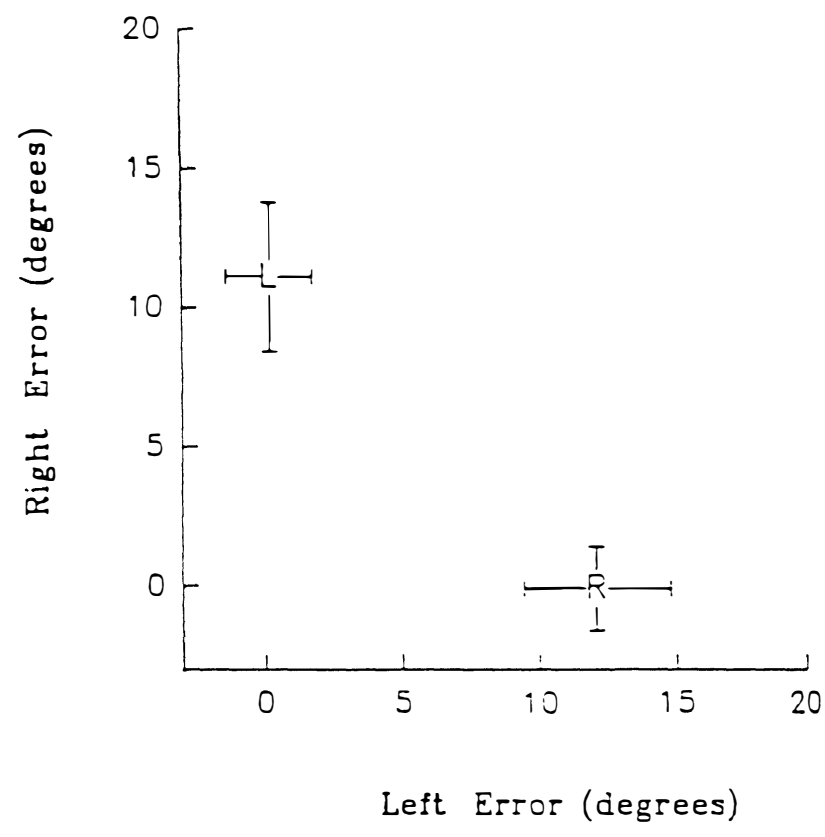

Fig. 8. The fixation positions of the left and right eye during monocular fixation by a monkey with essential infantile esotropia. During left eye fixation there is no error for the left eye and an error of about 11 degrees is apparent for the right eye. Similarly during right eye fixation there is no error for the right eye, but a 12 degree error for the left eye. The scatter in the two eyes (error bars in the horizontal and vertical directions) are not the same. The fixating eye always has relatively small amounts of scatter that fall within the range seen in normal monkeys. The non-fixating eye always has an abnormally large amount of scatter. This pattern of results was never seen for any monkeys except those with essential infantile esotropia.

results described here lead to the expectation that sensory input from only one eye should be providing input to these structures at any one moment in time, and that the eye which is providing the input will alternate over time.*

Additional quantitative studies of the fixation scatter in each eye of naturally strabismic monkeys have led us to discover other important properties of vergence control in strabismus. The pattern of scatter exhibited in monkeys with essential infantile strabismus was different from that seen in all other types of strabismus. This pattern is illustrated in Fig. 8. We used polarising filters arranged in such a manner that the monkey was able to view the fixation target with only one eye, but we were able to measure fixation simultaneously in both eyes, and record the amount of error relative to the fixation target. Plots of the mean amounts of error for each eye during monocular viewing with the left eye and with the right eye

\footnotetext{
* Recent electrophysiological findings in monkeys are consistent with this prediction. King and Zhou report that oculomotor neuron motor commands are encoded as a combination of left and right eye movements rather than conjugate and vergence movements (King WM, Zhou W. Encoding of eye movement parameters by burst-tonic neurons during disjunctive smooth pursuit and saccades. Soc Neurosci Abstr 1995;21:140).
} 
are shown in Fig. 8. It can be seen that when this monkey is fixating with its left eye, there is no error for the fixating eye, but approximately 11 degrees of error for the right eye. Similarly, during right eye viewing, the right eye is on target but the left eye exhibits about 12 degrees of error. This monkey has an esotropia and, as expected, the mean error values are the same under binocular viewing (results not shown) as shown here in Fig. 8 for monocular viewing.

The error bars around the symbols in Fig. 8 show the standard deviations of the scatter in eye position. Note that the error bars are not the same magnitude for both eyes. During left eye viewing the error bars are relatively small for the left eye and larger for the right eye. During right eye viewing, the scatter is larger for the left than for the right eye. Thus the amount of scatter is not related to anatomical left or right eye. Instead, it is related to the eye that is controlling fixation. This finding can be related to the model shown in Fig. 7 by stating that the eye which is controlling fixation always results in small scatter for the fixating eye. The larger amount of scatter is always associated with the eye being controlled by binocular sensory processing. This pattern of scatter is never seen in any of our monkeys with other types of strabismus, nor is it seen in normal monkeys. In monkeys with types of strabismus other than essential infantile, the amount of scatter varies according to which eye is fixating. If the preferred eye is being used for fixation then scatter is small in both eyes. If the animal is forced to fixate with its non-preferred eye, then scatter is large in both eyes. These scatter results where the magnitude of scatter is the same in both eyes can be modelled by adding noise to the sensory processing from the fixating eye. However, the results from the monkeys with essential infantile esotropia can only be modelled by adding noise at the level of binocular ('double eye') processing. These results are consistent with an old concept in the ophthalmological literature that goes back to the writings of Worth, namely that the essential infantile syndrome involves a congenital defect of the fusion faculty. ${ }^{33,34}$

\section{BINOCULARITY AND STRABISMUS FROM A DEVELOPMENTAL PERSPECTIVE}

Studies have been performed with infant monkeys to determine the sensitive period during which improper binocular stimulation will disrupt the development of the binocular visual system. As illustrated in Fig. 1 the relationships between sensory and motor binocular processing are reciprocal. Thus it is possible to disrupt binocular control of motor output in an infant and this leads to maldevelopment of binocular sensory processing. Likewise, if an infant monkey is deprived of binocular sensory processing during early development, a strabismus will develop. ${ }^{35-37}$

In some cases, a strabismus that is present during early development will also lead to an amblyopia. The sensitive period during which introduction of an experimental strabismus leads to amblyopia in monkeys lasts about 12 weeks. ${ }^{35}$ The loss of visual acuity is not immediate, but first appears several weeks after initiation of the experimental strabismus. Two major theoretical positions have been proposed regarding the causal factors that operate to allow a strabismus to produce an amblyopia. ${ }^{35}$ The first states that strabismic amblyopia is caused by mild blur in the deviated eye. The argument is essentially that in a normal infant the foveae of both eyes would be expected to receive equal amounts of high spatial frequency stimulation as the infant repeatedly scanned across contours in the visual environment. On the other hand, a strabismic infant with a fixation preference will optimise spatial frequency stimulation only for the preferred eye. It would also be expected that a strabismic infant with an equally alternating fixation pattern would receive equal amounts of high spatial frequency stimulation in both eyes. The stimulation will just be received sequentially from each eye in turn instead of simultaneously in both eyes as occurs in a normal infant. Observations on experimentally strabismic infant monkeys reveal that animals with a freely alternating fixation pattern usually do not develop amblyopia, whereas those that consistently fixate with only one eye eventually develop an amblyopia in the opposite eye.

The second position with regard to the causes of

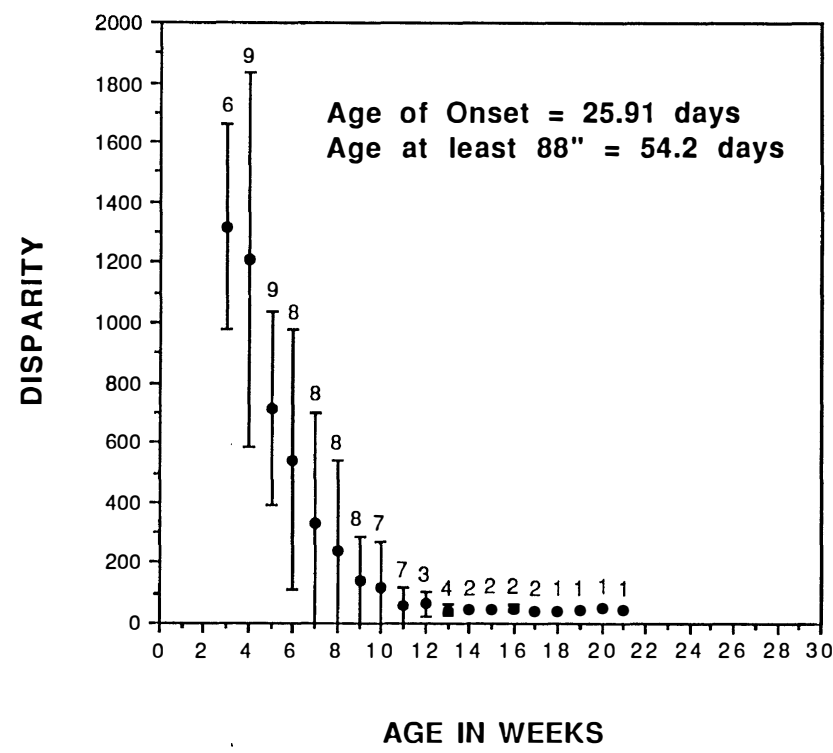

Fig. 9. The time course for the development of stereoacuity in infant monkeys. The average age of onset for the largest disparity we used during testing (1700 seconds of arc) was 26 days after birth. Stereoacuity values had improved to a mean level of 88 seconds by 54 days of age. 


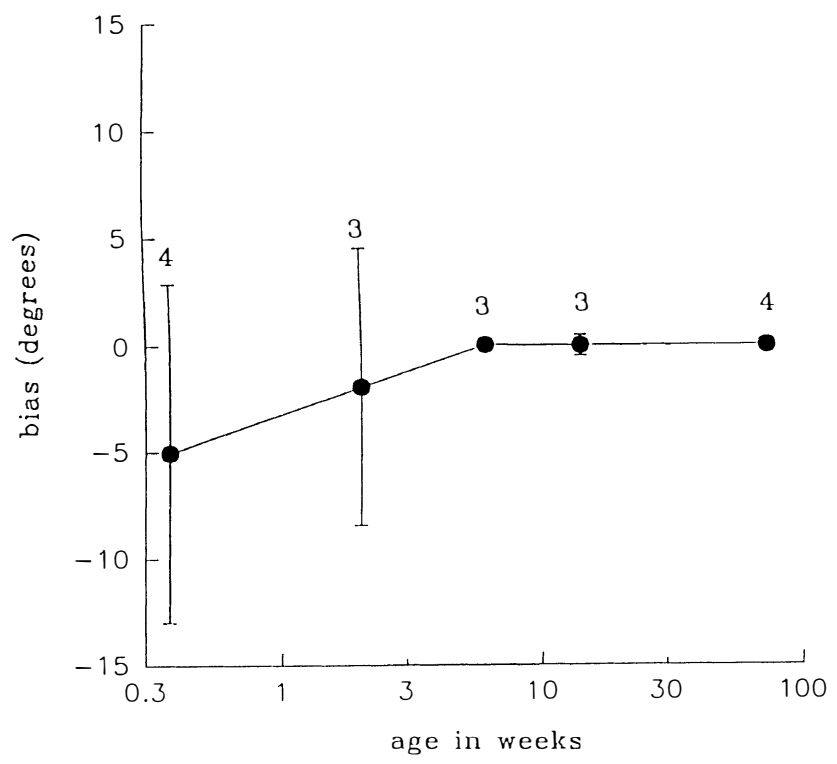

Fig. 10. The time course for development of eye alignment in infant monkeys. ${ }^{46}$ The mean amount of deviation ('bias') is shown at every age tested, with positive values designating esodeviations and negative values designating exodeviations. At the youngest ages monkeys tend to be exotropic, but also exhibit increased variability. This is primarily due to the fact that the infants over-converge and become esotropic when viewing near targets, but are otherwise usually exotropic.

strabismus amblyopia stresses the importance of binocular interactions such as interocular suppression. ${ }^{34}$ The rationale is that strabismus leads to an abnormal binocular input in the form of diplopia and confusion. Sensory adaptations such as binocular suppression then develop to alleviate the adverse effects of this abnormal input. Under conditions in which an infantile or acquired strabismus is left uncorrected during the developmental sensitive period, binocular suppression can become converted into a permanent condition of inhibition of one eye's pathways that persists even under monocular viewing conditions. Evidence in support of this position comes from studies that involve enucleation of the fellow eye. For example, enucleating the fellow eye of a monkey with strabismic amblyopia results in behavioural improvements in the functional capacity of the amblyopic eye, whereas enucleation does not lead to similar improvement in monkeys with stimulus-deprivation amblyopia. ${ }^{38,39}$

Another binocular function that fails to develop properly in monkeys with an experimentally induced strabismus is stereopsis. The onset for stereopsis in normal infant monkeys occurs at about 3-4 weeks of age (Fig. 9). ${ }^{40}$ This capacity never develops in infant monkeys with a strabismus or in infant monkeys in which alternating occlusion is used to prevent normal binocular stimulation during infancy. However, a somewhat surprising finding is that if binocular deprivation that is initiated at birth is terminated at

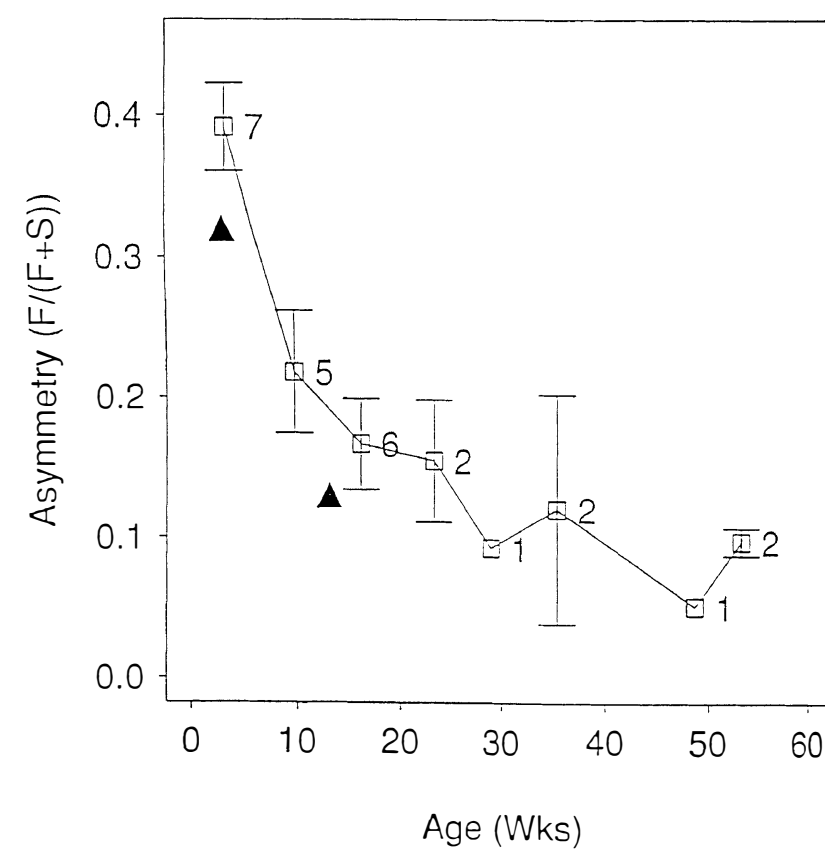

Fig. 11. The squares demonstrate the development from asymmetrical processing of motion to symmetrical processing of motion as measured with visually evoked potentials $(V E P)$. The asymmetry is quantified as the ratio of the Asymmetrical $(F)$ component of the VEP to the combined Asymmetrical and Symmetrical $(S)$ components. Numbers next to each data point designate the number of monkeys tested at each stage. The triangles indicate the ages at which stereopsis has its initial onset and when stereoacuity matures to near adult levels (see Fig. 9). The developmental time period during which motion processing becomes symmetrical overlaps with the period during which stereopsis emerges. ${ }^{47}$

3-4 weeks of age, which is the time when stereopsis should be coming in, then stereoacuity still develops, although with a delay of a few weeks. ${ }^{41}$

Another deficit that arises from an early onset infantile strabismus is motion asymmetry. ${ }^{42,43}$ This motion asymmetry only occurs following binocular visual deprivation during a very early sensitive period prior to the onset of stereopsis. Similarly, a permanent strabismus is only produced by binocular sensory deprivation when the deprivation conditions are instituted at a very early age. ${ }^{36,44}$ This suggests that there is a dramatic reorganisation of the brain that takes place at about 3 weeks of age in the infant monkey such that binocular deprivation that occurs prior to this reorganisation has effects that are more severe than, and perhaps even qualitatively different from, the effects of later onset deprivation.

Tyschsen ${ }^{45}$ developed a theory of the aetiology of strabismus based on a cortically based asymmetry of sensory motion processing and control of smoothpursuit eye movements. In his scheme, sensorydependent motor responses controlling eye movements can introduce in the infant a chronic eye misalignment. Since fusion obviously depends on proper eye alignments, and since human newborns 
usually start out exotropic, binocular vision cannot develop until the visual axes converge sufficiently to make fusion possible. Tychsen argues that the natural nasalward motion asymmetry observed in infants actually serves to 'pull' the eyes together from the exotropic position. When the eyes are brought to converge on an object, a fusion signal could in principle generate a negative feedback to prevent the eyes from over-converging. If this signal fails to take place the nasal bias would continue to impress its effect upon motor movements and the eyes would consequently continue to turn inward further, eventually passing their fusional range and leading to strabismus.

Some of our results from monkeys that are in general agreement with Tychsen's theory. The eyes of neonatal monkeys are not aligned or stable (see Fig. 10). The misalignments tend to be in the exotropic direction, although the neonates tend to over-converge and become esotropic during near fixation which leads to a high amount of variability. If one looks at the time course over which the eyes become aligned in conjunction with the time course over which motion symmetry matures and over which stereopsis develops (Figs. 9-11), a nice progression can be seen which is consistent with Tychsen's basic scheme. The eyes start out variable but in a predominantly exotropic condition. Then they start converging during a period when there is a high motion asymmetry present. At about the time stereopsis develops, eye alignment is becoming orthotropic and soon thereafter the motion asymmetry stops. Any disruption of normal binocular processing (on either the motor or the sensory side) during this very early sensitive period can lead to permanent strabismus and motion asymmetry in addition to lack of stereopsis.

I thank Mrs Jean Torbit for assistance in preparing this manuscript for publication. Preparation of this paper and support for some of my own research described here have been provided by NIH grants EY-05975 and RR-00165, and by a URC grant from Emory University.

Key words: Binocularity, Binocular function, Depth perception, Primates, Stereovision, Strabismus, Visual development.

\section{REFERENCES}

1. Marr D, Poggio T. A computational theory of human stereo vision. Proc Soc Lond B 1979;204:301-28.

2. Longuet-Higgins FRS. The reconstruction of a plane surface from two perspective projections. Proc R Soc Lond B 1986;227:399-410.

3. Julesz B. Stereoscopic vision. Vision Res 1986; 26:1601-12.

4. Lehky SR, Sejnowski TJ. Neural model of stereoacuity and depth interpolation based on a distributed representation of stereo disparity. J Neurosci 1990; 10:2281-99.

5. Blake R, Wilson HR. Neural models of stereoscopic vision. Trends Neurosci 1991;14:445-52.
6. Gibson JJ. The ecological approach to visual perception. Boston: Houghton Mifflin, 1979.

7. Lee DN. The optic flow field: the foundation of vision. Phil Trans R Soc Lond B 1980;290:169-79.

8. Buxton BF, Buxton H. Monocular depth perception from optical flow by space time signal processing. Proc R Soc Lond B 1983;218:27-47.

9. Koenderink JJ. Optic flow. Vision Res 1986;26:161-80.

10. Polyak S. The vertebrate visual system. Chicago: University of Chicago Press, 1957.

11. Walls GL. The vertebrate eye and its adaptive radiation. New York: Hafner, 1963. (Reprinted from the Cranbrook Institute of Science, Bloomfield Hills, MI, 1942.)

12. Ingle D. A possible behavioral correlate of delayed retinal discharge in Anurans. Vision Res 1971;11: 167-8.

13. Ingle D. Evolutionary perspectives on the function of the optic tectum. Brain Behav Evol 1973;8:211-3.

14. Ingle D. Functions of subcortical visual systems in vertebrates and the evolution of higher visual mechanisms. In: Cronly-Dillon JR, general editor. Vision and visual dysfunction, vol 2, Evolution of the eye and visual system. Boca Raton: CRC Press, 1991:152-64.

15. Helmholtz $\mathrm{H}$, von. Handbuch der physiologischen Optik. Hamburg: Voss, 1866. (Transl from German by Southall JPC. 1924/1925. Republished as one volume, New York: Dover, 1962.)

16. Regan D. Depth from motion and motion-in-depth. In: Cronly-Dillon JR, general editor. Vision and visual dysfunction, vol 3, Binocular vision. Boca Raton: CRC Press, 1991:137-69.

17. Sobel EC. Depth perception by motion parallax and paradoxical parallax in the locus. Naturwissenschaften 1990;77:241-3.

18. Poggio GF, Talbot WH. Mechanisms of static and dynamic stereopsis in foveal cortex of rhesus monkey. J Physiol (Lond) 1981;315:469-92.

19. Shimojo S, Silverman GH, Nakayama K. An occlusionrelated mechanism of depth perception based on motion and interocular sequence. Nature 1988; 333:265-7.

20. Boothe RG. Experimentally induced and naturally occurring munkey models of human amblyopia. In: Berkley MA, Stebbins WC, editors. Comparative perception, vol 1, Basic mechanisms. New York: Wiley, 1990: 461-86.

21. Boothe RG, Quick MW, Joosse MV, Abbas MA, Anderson DC. Accessory lateral rectus orbital geometry in normal and naturally strabismic monkeys. Invest Ophthalmol Vis Sci 1990;31:1168-74.

22. Beverly KI, Regan D. Evidence for the existence of neural mechanisms selectively sensitive to the direction of movement in space. J Physiol (Lond) 1973;235: $17-29$.

23. Beverly KI, Regan D. The relationship between discrimination and sensitivity in the perception of motion in depth. J Physiol (Lond) 1975;249:387-98.

24. Richards W. Response functions for sine- and squarewave modulations of disparity. J Opt Soc Am 1972;62: 907-11.

25. Jampolsky A. Unequal visual inputs and strabismus management: a comparison of human and animal strabismus. In: Symposium on strabismus: Transactions of the New Orleans Academy of Ophthalmology. St Louis: Mosby, 1978:358-492.

26. Kiorpes L, Boothe R. Naturally occurring strabismus in monkeys (Macaca nemestrina). Invest Ophthalmol Vis Sci 1981;20:257-63. 
27. Kiorpes L, Boothe R, Carlson M, Alfi D. Frequency of naturally occurring strabismus in monkeys. J Pediatr Ophthalmol Strabismus 1985;22:60-4.

28. Quick MW, Eggers H, Boothe RG. Natural strabismus in monkeys: convergence errors assessed by cover test and photographic methods. Invest Ophthalmol Vis Sci 1992;33:2986-3004.

29. Quick MW, Newbern JD, Boothe RG. Natural strabismus in monkeys: accommodative errors assessed by photorefraction and their relationship to convergence errors. Invest Ophthalmol Vis Sci 1994;35: 4069-79.

30. Hering E. The theory of binocular vision. In: Bridgeman B, Stark L, editors. The theory of binocular vision. New York: Plenum Press, 1977.

31. Judge SJ. How is binocularity maintained during convergence and divergence? Eye 1996;10:172-6.

32. Gamlin PDR, Yoon K, Zhang H. The role of cerebroponto-cerebellar pathways in the control of vergence eye movements. Eye 1996;10:167-71.

33. Lang J. Strabismus. Thorofare, NJ: Slack, 1984.

34. Noorden GK, von. Binocular vision and ocular motility: theory and management of strabismus. St Louis: CV Mosby, 1985.

35. Boothe RG. Amblyopia. In: Albert DM, Jakobiec FA, editors. Principles and practice of ophthalmology: basic sciences. Philadelphia: WB Saunders, 1993.

36. Quick M, Tigges M, Gammon J, Boothe RG. Early abnormal visual experience induces strabismus in infant monkeys. Invest Ophthalmol Vis Sci 1989; 30:1012-7.

37. Crawford MLJ, Harwerth RS, Chino YM, Smith EL III. Binocularity in prism-reared monkeys. Eye 1996; 10:161-6

38. Harwerth RS, Smith EL, Duncan GC, et al. Effects of enucleation of the fixating eye on strabismic amblyopia in monkeys. Invest Ophthalmol Vis Sci 1986;27:246-54.

39. Harwerth RS, Smith EL, Crawford MLJ, et al. Effects of enucleation of the nondeprived eye on stimulus deprivation amblyopia in monkeys. Invest Ophthalmol Vis Sci 1984;25:10-8.

40. O'Dell CD, Quick MW, Boothe RG. The development of stereoacuity in infant rhesus monkeys. Invest Ophthalmol Vis Sci (ARVO Suppl) 1991;32:1044.

41. Jampolsky A, Brown RJ, Boothe RG, Wilson JR, Tigges M, Norcia AM, Fernandes A. Delay of stereoacuity development in monkeys by full time alternate occlusion. Invest Ophthalmol Vis Sci (ARVO Suppl) 1993;34:1188.

42. Brown RJ, Norcia AM, Hamer RD, Wilson JR, Boothe $\mathrm{RG}$. Development of motion processing mechanisms in monkey and human infants. Invest Ophthalmol Vis Sci (ARVO Suppl) 1993;34:1356.

43. Norcia AM. Abnormal motion processing and binocularity. Eye 1996;10:259-65.

44. Boothe RG, Gong W. Development of binocular alignment in normal and visually deprived monkeys. Invest Ophthalmol Vis Sci (ARVO Suppl) 1992;33:871.

45. Tychsen L. Motion sensitivity and the origins of infantile strabismus. In: Simons K, editor. Infant vision: basic and clinical research. New York: Oxford University Press.

46. Quick MW. Experimentally induced monkey models of infantile strabismus. Doctoral Dissertation, Emory University Graduate School, Atlanta, Georgia, USA, 1992.

47. Brown RJ. Deprivation of binocular vision and consequences on motion perception. Master's Thesis, Emory University Graduate School, Atlanta, Georgia, USA, 1995. 